MODELING, IDENTIFICATION AND CONTROL, 1990, VOL. 11, NO. 3, 155-167

doi:10.4173/mic.1990.3.3

\title{
An algorithm for design of decentralized suboptimal controllers with a specified structure
}

\author{
DAVID DI RUSCIO† and JENS G. BALCHEN
}

Keywords: Control system design, decentralized control, linear optimal control, optimization

In this paper we present a method for the design of controllers with a specified arbitrary structure for linear multivariable time invariant systems. Both decentralized controllers as well as feedback from a reduced state vector can be designed by this method. The controller will become optimal in the sense that it yields a minimum of a quadratic cost criterion and suboptimal in the sense that it yields a higher value of this criterion than a controller without restrictions. The algorithm makes it possible to specify a stability margin on the feedback system. This means that the feedback system will have eigenvalues located to the left of a certain line $(-\alpha)$ in the complex plane. The unknown parameters of the controller are collected in a parameter vector. The algorithm is based upon a modified Newton-method for searching towards the 'optimal' parameter vector. The algorithm ensures that the closed loop system is stable at any iteration in the case of an initially stable plant, and after the final iteration in the case of an initially unstable plant.

\section{Introduction}

Levine and Athans (1970) gave a necessary condition for 'optimality' when a feedback is established from a reduced state vector for a linear time invariant system. This result involves the solution of a set of nonlinear matrix equations which is a difficult task. This same problem is dealt with in Solheim (1976). The above method does not allow specification of a decentralized control structure.

Kosut (1970) generalizes the result into two algorithms for the determination of a suboptimal decentralized controller. Suboptimal here means that the controller does not necessarily give a minimum of the criterion. Furthermore, neither of the methods presented by Kosut guarantee that the closed loop system will be stable. This must be checked explicitly. Geromel and Bernussou (1979) present an algorithm for the design of suboptimal decentralized controllers based upon a search method of 'feasible directions'. This algorithm is based upon the solution of two linear matrix Liapunov equations at each iteration, and needs to be initialized with a stabilizing control. An advantage of this method is that it guarantees a stable solution at each iteration. However, the algorithm converges very slowly because is based upon searching along a line in the gradient direction. It also turns out that the algorithm solves an unnecessary problem, namely the determination of the gradient of the criterion for the case of no restrictions on the controller and thereafter sets the gradients in the undesired directions to zero. Geromel and Bernussou also do not indicate any possibility for including a specified stability margin in the criterion.

Received August 11990

$\dagger$ Division of Engineering Cybernetics, Norwegian Institute of Technology, N-7034 Trondheim, Norway. 
The algorithm to be presented here allows us to design 'optimal' decentralized controllers with specified structures. It is only necessary to solve one matrix Liapunov equation at each function evaluation, which guarantees the stability. Furthermore, the algorithm does not involve any unnecessary computation because the search for the unknown parameter vector is done in the gradient direction.

If an initial stabilizing controller exists, for example the zero control if the nominal system is stable, a method for unconstrained optimization is employed in the algorithm. In this case the closed loop system is guaranteed to be stable at each iteration and the solution converges to a local minimum. In the case that the system is initially unstable, and an initial stabilizing controller does not exist, constraints to ensure that the final closed loop system is stable are added to the problem, and a method for constrained optimization is employed.

The specification of a certain margin of stability for the resulting closed loop system is based on Anderson and Moore (1971).

Section 2 will define the problem. Section 3 describes the method of inclusion of the stability margin in the criterion. Section 4 presents a new method for the deisgn of linear controllers with a specific arbitrary structure. Section 5 deals with the problem of tuning controllers, which may possess some structure, for initial unstable plants. In Section 6 some examples are presented, one on the use of the algorithm for the design of controllers for a binary distillation column and another for tuning controllers for an initially unstable plant.

\section{Problem definition}

Assume a time invariant linear system of the form

$$
\dot{\boldsymbol{x}}=A \boldsymbol{x}+B \boldsymbol{u}
$$

and the quadratic objective functional

$$
J=\int_{t_{1}}^{\infty}\left(x^{\mathrm{T}} Q x+u^{\mathrm{T}} P u\right) d t
$$

where $\boldsymbol{x}$ is an $\boldsymbol{n}$-dimensional state vector, $\boldsymbol{u}$ is an $\boldsymbol{r}$-dimensional control input vector, $\mathbf{A}$ and $\mathbf{B}$ are constant matrices of appropriate dimensions and $\mathbf{Q}$ and $\mathbf{P}$ are $n \times n$ nonnegative and $r \times r$ positive definite weighting matrices, respectively.

The problem is to design a controller matrix $\mathbf{G}$ of dimension $r \times n$ so that the system (1) gets the linear feedback

$$
u=G x
$$

and so that the following requirements are satisfied

$\mathbf{G}$ has an arbitrary specified structure (diagonal, blockdiagonal, sparse etc.).

$\mathbf{G}$ is determined so that the quadratic performance criterion (2) is minimized.

$\mathbf{G}$ is determined so that the system (1) with controller (3) gets a specified stability margin.

The algorithm will make it possible to determine decentralized P, PI and PID controllers for multivarible processes in a systematic manner. Furthermore the determination of feedback from a reduced state vector is made possible. This means that the controller matrix $\mathbf{G}$ has certain rows with only zeros. 
If an $m$-dimensional vector $y$ of measurements is given by the linear equation

$$
\boldsymbol{y}=D \boldsymbol{x}
$$

then the problem above may be formulated as the output feedback design problem. The linear feedback is in this case given by

$$
\boldsymbol{u}=G_{y} y=G_{y} D x
$$

An alternative criterion to (2), to be minimized subject to (1) and (5), is

$$
J=\int_{t_{1}}^{\infty}\left(x^{\mathrm{T}} D^{\mathrm{T}} Q_{y} D x+u^{\mathrm{T}} P u\right) d t
$$

This problem is the same as that in (2) and (3) substituted for $Q \rightarrow D^{\mathrm{T}} Q_{y} D$ and $G \rightarrow G_{y} D$.

\section{Specification of stability margin}

We assume that the overall system (with the controller) is specified to have a stability margin such that all eigenvalues have real parts less than $-\alpha$. Therefore we modify the criterion (2) as suggested by Anderson and Moore (1971) to

$$
J=\int_{t_{1}}^{\infty} \exp (2 \alpha t)\left(x^{\mathrm{T}} Q x+u^{\mathrm{T}} P u\right) d t
$$

Furthermore we introduce

$$
\overline{\boldsymbol{x}}=\exp (\alpha t) x \quad \text { and } \quad \overline{\boldsymbol{u}}=\exp (\alpha t) u
$$

Now we have a modified problem of the same form as that introduced in Section 2

$$
\begin{aligned}
& \dot{\bar{x}}=(A+\alpha I) \bar{x}+B \bar{u} \\
& J=\int_{t_{1}}^{\infty}\left(\bar{x}^{\mathrm{T}} Q \overline{\boldsymbol{x}}+\overline{\boldsymbol{u}}^{\mathrm{T}} P \bar{u}\right) d t
\end{aligned}
$$

By solving this LQ optimal control problem, (determine a controller for (9) so that the closed loop system is stable and (10) is minimized), we obtain a linear feedback controller of the form

$$
\overline{\boldsymbol{u}}=\boldsymbol{G} \overline{\boldsymbol{x}}
$$

which is seen to be a linear feedback controller of the form $\boldsymbol{u}=\boldsymbol{G x}$ for the original system, and which yields the desired stability margin. This means that when $\mathbf{G}$ is determined for the modified problem in (9) and (10), the system in (1) will converge faster than an exponential with time constant $1 / \alpha$ because

$$
\boldsymbol{x}=\exp (-\alpha t) \overline{\boldsymbol{x}}
$$

in which $\overline{\boldsymbol{x}}$ is a stable trajectory.

\section{Solution algorithm}

Independent of how $\mathbf{G}$ is determined the following must be satisfied (Bryson and Ho 1969)

$$
\min _{w} J=\exp \left(2 \alpha t_{1}\right) x^{\mathrm{T}}\left(t_{1}\right) R x\left(t_{1}\right)=\exp \left(2 \alpha t_{1}\right) \operatorname{tr}\left(x\left(t_{1}\right) x^{\mathrm{T}}\left(t_{1}\right) R\right)
$$


where $R$ must satisfy the equation

$$
(A+\alpha I+B G)^{\mathrm{T}} R+R(A+\alpha I+B G)+Q+G^{\mathrm{T}} P G=0
$$

The parameter vector $\theta$ is defined as the unknown nonzero elements in $\mathbf{G}$. In addition comes a matrix $F$ which specifies the structure. This is described in the definitions below.

Definition 1. The elements in the $r \times n$ dimensional structure matrix $\mathbf{F}$ is defined by:

$$
f_{i j}= \begin{cases}1 & \text { if } g_{i j} \neq 0 \\ 0 & \text { if } g_{i j}=0\end{cases}
$$

We note that $F$ satisfies $F x G=G$ where $x$ denotes element by element matrix multiplication.

Definition 2. Given a general $r \times n$ matrix G. The $p$-dimensional parameter vector $\theta$ can be formed from the column vectors of $\mathbf{G}$ given a matrix structure defined by $\mathbf{F}$. We will introduce the column structure string (css) operator in order to effect such transformations:

$$
\theta=\operatorname{css}(G, F)
$$

In order to effect the transformation from the vector $\theta$ to a matrix $\mathbf{G}$, when a matrix structure is defined by the matrix $\mathbf{F}$, we introduce the inverse column structure string operator:

$$
G=\operatorname{css}^{-1}(\theta, F)
$$

Let $p=\operatorname{dim} \theta$ which is equal to the number of nonzero elements in $\mathbf{F}$. The problem of determining the optimal feedback matrix $\mathbf{G}$ is thereby converted to that of determining $\theta$ from the following optimization problem (where for the sake of simplicity $t_{1}=0$ ).

$$
\begin{gathered}
(A+\alpha I+B G)^{\mathrm{T}} R+R(A+\alpha I+B G)=-\left(Q+G^{\mathrm{T}} P G\right) \\
\min _{\theta} J=\min _{G} J=\operatorname{tr}\left(x(0) x^{\mathrm{T}}(0) R\right)
\end{gathered}
$$

It is seen that the initial state $x(0)$ appears in (19) which means that the optimal criterion value depends on the initial state. If we do not impose restrictions on the controller, the optimal solution $\mathbf{G}$ of (19) will be independent of $\boldsymbol{x}(0)$. However, when the controller is to have a special structure, the optimal solution $\mathbf{G}$ will depend on the initial state. This problem can be avoided by regarding $\boldsymbol{x}(0)$ as a stochastic variable and assuming

$$
E(x(0))=0, \quad E\left(x(0) x^{\mathrm{T}}(0)\right)=I
$$

For a given $\mathbf{G},(18)$ will become a matrix Liapunov equation with $\mathbf{R}$ as unknown. If $\mathbf{G}$ is such that $(A+\alpha I+B G)$ is a stable matrix and $\left(Q+G^{\mathrm{T}} P G\right)$ is positive definite, then there is a guarantee that the solution $\mathbf{R}$ of (18) will be positive definite and symmetric.

The symmetry is guaranteed because the right-hand side of (18) is symmetric and the positive definiteness is known from Liapunov's second method. Note that when the control input weight matrix $P=P^{\mathrm{T}}>0$ then $G^{\mathrm{T}} P G \geqslant 0$ and that $Q$ is assumed to be nonnegative. This implies that $\left(Q+G^{\mathrm{T}} P G\right)$ is at least non-negative. Note also that for a solution of the Liapunov equation to exist, the eigenvalues $\lambda$ of $(A+\alpha I+B G)$ must satisfy $\lambda_{i}+\lambda_{j} \neq 0, i, j=1, \ldots, n$ and that the solution is not unique when $(A+\alpha I+B G)$ is singular. This means that a unique solution exists when $(A+\alpha I+B G)$ is stable. 
The matrix Liapunov Equation (18) is linear in R for a given G. In order to determine the value of the functional $J(\theta)$ in (19), R must be determined from (18) and this is therefore an important part of the optimization problem. A special study has been done to find the best method for solving matrix Liapunov equations (Di Ruscio 1988). In this work we used the method by Chen (1968). However, Chen's method has little to recommend it from a numerical point of view compared to applying the Bartels-Stewart algorithm (1972) or the algorithm by Golub et al. (1979).

Introducing $k$ as an index of iteration, the following algorithm is developed for determining the value of the functional $J\left(\theta_{k}\right)$ for a given $\boldsymbol{\theta}_{k}$.

\section{Algorithm 4.1, computing $J\left(\theta_{k}\right)$}

(1) Given a parameter vector $\theta_{k}$. Transform $\theta_{k}$ to the feedback $G_{k}$ with feedback structure given by $\mathbf{F}$, i.e.

$$
G_{k}=\operatorname{css}^{-1}\left(\theta_{k}, F\right)
$$

(2) Determine $R_{k}$ for a given $G_{k}$ which is a solution to the Liapunov equation.

$$
\left(A+\alpha I+B G_{k}\right)^{\mathrm{T}} R_{k}+R_{k}\left(A+\alpha I+B G_{k}\right)=-\left(Q+G_{k}^{\mathrm{T}} P G_{k}\right)
$$

(3) Determine the value of the functional

$$
J\left(\theta_{k}\right)=\operatorname{tr}\left(R_{k}\right)
$$

Now a search technique for the determination of the optimal parameter vector $\theta$ can be employed (Schittkowski 1984, Wolfe 1978). The optimization problem in (18) and (19) is nonlinear in $\theta$. We now propose to employ a Newton method where the gradient of $J(\theta)$ with respect to $\theta$ and the Hessian matrix are determined numerically by perturbation of $\theta$. The algorithm is described in detail in Wolfe (1978). A description is given below.

\section{Algorithm 4.2, solution procedure}

(1) Specify an initial parameter vector $\theta_{0}$. Determine the feedback matrix by the transformation $G_{0}=\operatorname{css}^{-1}\left(\theta_{0}, F\right)$. The iteration must be initialized with a choice of $G_{0}$ such that $\left(A+\alpha I+B G_{0}\right)$ is stable.

(2) Determine the gradient $g_{k}=\partial J / \partial \theta_{k}$ numerically. Values of functional $\mathbf{J}$ are determined by Algorithm 4.1. See also comments below.

(3) Test convergence. If all elements of $\boldsymbol{g}_{\boldsymbol{k}}$ are less than a specified small value the gradient search is terminated.

(4) Determine the Hessian matrix $H_{k}=\partial^{2} J / \partial \theta_{k}^{2}>0$.

(5) Determine search direction $\boldsymbol{p}_{k}$ from $H_{k} p_{k}=-g_{k}$. Update the parameter vector from $\theta_{k+1}=\theta_{k}+\alpha_{k} p_{k}$ where $\alpha_{k}$ is a line search parameter determined such that $0 \leqslant J\left(\theta_{k+1}\right)<J\left(\theta_{k}\right)$ is satisfied. Return to item 2 .

The elementary form of Newton's method can run into problems if the following appears:

$H_{k}^{-1}$ exists and is positive definite but $p_{k}$ is so large that $J_{k+1}>J_{k}$.

The direction $p_{k}$ is orthogonal to $g_{k}$.

$H_{k}^{-1}$ exists but is not positive definite.

$H_{k}^{-1}$ does not exist. 
The actual algorithm which is implemented is modified to handle this situation. (For a detailed description see Wolfe (1978) ch. 3.3).

Item 1 in the algorithm assumes an initial value of $\theta$ which stabilizes the closed loop system. If the process (without closed-loop control) is stable the initial value of $\theta$ can be set to zero. If the nominal system is unstable, constraints to ensure that the final closed loop system is stable may be included in the algorithm. This problem is further discussed in Section 5.

Item 2 in the algorithm above is the determination of the gradient $g_{k}=\partial J / \partial \theta_{k}$. This is done numerically from the expression

$$
\frac{\partial J}{\partial \theta_{k}} \approx \frac{J\left(\theta_{k}+h e_{j}\right)-J\left(\theta_{k}-h e_{j}\right)}{2 h}, j=1, \ldots, p
$$

where $e_{j}$ is an $p$-dimensional unit vector with 1 in position $j$ and the rest of the elements $\mathbf{0} . \boldsymbol{h}$ is a small (computer dependent) quantity. The values of the functional $\mathbf{J}$ are determined as described above. The Hessian matrix in item 3 is determined numerically from the gradient calculations. This is described in Wolfe (1978) ch. 3.5 p. 109.

In item 5 it is noted that the functional value must satisfy $J(\theta) \geqslant 0$. The reason for this is simply because

$$
J(\theta)=\operatorname{tr}(R)=\operatorname{tr}\left(S^{\mathrm{T}} \Lambda S\right)=\operatorname{tr}(\Lambda)>0
$$

where $\Lambda$ is a diagonal eigenvalue matrix, $S$ is an orthogonal eigenvector matrix and $R$ is positive definite. The case $J=0$ corresponds to the case with $Q=0$ and no control.

The suboptimal cost may be compared with the expected optimal cost by the performance index

$$
\gamma=\frac{\operatorname{tr}(R)}{\operatorname{tr}\left(R_{\text {opt }}\right)} \geqslant 1
$$

where $\boldsymbol{R}_{\mathrm{opt}}$ is the optimal solution to the problem, i.e. the case without constraints on the controller structure. The suboptimal cost can also be compared with any other cost.

\section{The output feedback design and the stabilization problem}

In Section 4 we have solved the problem of designing a linear optimal constant feedback from a (reduced) state vector, where the feedback matrix $\mathbf{G}$ may have some structure.

Suppose that one now introduces the constraints that the control $u$ be generated via output linear feedback, as stated in Section 2, Equations (4), (5) and (6). The solution to this problem is the same as that in Section 4. Substituting $G \rightarrow G_{y} D$ and $Q \rightarrow D^{\mathrm{T}} Q_{y} D$ in Equation (14) gives

$$
\left(A+\alpha I+B G_{y} D\right)^{\mathrm{T}} R+R\left(A+\alpha I+B G_{y} D\right)=-\left(D^{\mathrm{T}} Q_{y} D+\left(G_{y} D\right)^{\mathrm{T}} P G_{y} D\right)
$$

If an initial stabilizing matrix $G_{y}$ is available, then the optimization algorithm presented in Section 4 is sufficient to determine the optimal parameter vector $\theta$.

If the system is open-loop unstable, then we have two problems to deal with, one of existence and one of the construction of a stabilizing feedback matrix. The existing problem has a yes/no answer. This problem is dealt with by, among others, Anderson et al. (1975), Anderson and Scott (1977). If a construction algorithm fails, it indicates non-existence of a stabilization controller. 
The construction problem may be solved by adding some constraints to the optimization problem in Section 4 to ensure that the final closed loop matrix $A+B G$ or $A+B G_{y} D$ is stable. Routh or Hurwitz conditions may be used to determine constraints from the closed loop matrix characteristic polynomial, however for this problem it seems to be more elegant to restrict the solution $\mathbf{R}$ of (27) to be positive semi-definite. If a non-negative $\mathbf{R}$ is found, and the system is detectable, then the closed loop system is stable. This is due to Liapunov's second method.

Constraints may be set on the eigenvalues of $\mathbf{R}$, however it may be better with determinant (Sylvester) conditions. In addition we suggest to specify simple bounds on the optimal controller parameter vector $\theta$. Then we have the following optimization problem.

$$
\min _{\boldsymbol{\theta}} J=\min _{G_{\boldsymbol{y}}} J=\operatorname{tr}(R)
$$

subject to inequality constraints

$$
c_{i}=\operatorname{det}\left(R_{i i}\right) \geqslant 0, \quad i=1, \ldots, n
$$

and fixed bounds

$$
\theta_{i}^{\min } \leqslant \theta_{i} \leqslant \theta_{i}^{\max }, \quad i=1, \ldots, p
$$

where $\mathbf{R}$ is the solution to the linear matrix Liapunov equation (27).

Two well known optimization algorithms, NPSOL by (Gill et al. 1986) and NLPQ by (Schittkowski, 1984) may be used to solve the problem. In this work we use NLPQ, which has been found to be reasonable.

A numerical example of the design problem of an unstable plant is given in Section 6.2 .

\section{Numerical examples}

\subsection{Example 1, distillation column}

In this example we wish to use the algorithm to determine a multivariable PIcontroller for a binary distillation column. We impose the restriction that the only states available as measurements for feedback are the compositions at the top $\left(x_{8}\right)$ and the bottom $\left(x_{1}\right)$. For comparison we will also study the system under control when all states (compositions at all trays) are available. Data for the columns are given in Appendix A. The control structure is shown in the block diagram, Figure (1), where $H_{u}$ is a symbol for the plant (model).

If only the composition dynamics are considered a linearization of the non-linear model (see e.g., Di Ruscio 1987) will give the following linear model

$$
\begin{gathered}
\dot{x}=A x+B u+C v \\
y=D x
\end{gathered}
$$

where A, B and C are given in Appendix A. A state space model for the multivariable PI-controller will be

$$
\begin{gathered}
\dot{z}=D x \\
u=G_{1} y+G_{2} z=G_{1} D x+G_{2} z
\end{gathered}
$$




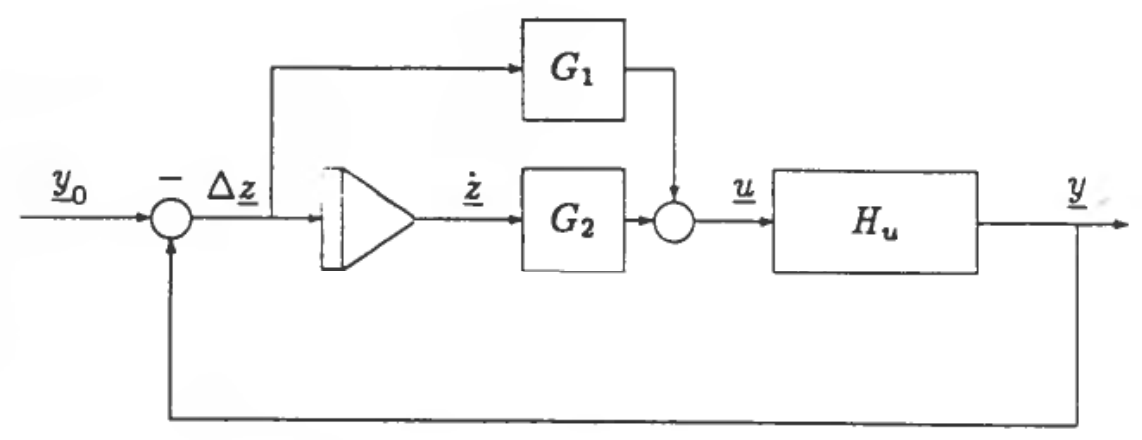

Figure 1. System with multivariable PI-controller.

In the augmented state-based form this will become

$$
\begin{gathered}
{\left[\begin{array}{c}
\dot{\boldsymbol{x}} \\
\dot{z}
\end{array}\right]=\left[\begin{array}{ll}
A & 0 \\
D & 0
\end{array}\right]\left[\begin{array}{l}
\boldsymbol{x} \\
z
\end{array}\right]+\left[\begin{array}{l}
B \\
0
\end{array}\right] u+\left[\begin{array}{l}
C \\
0
\end{array}\right] v} \\
u=\bar{G}\left[\begin{array}{l}
x \\
z
\end{array}\right]
\end{gathered}
$$

where $\bar{G}=\left[\begin{array}{ll}G_{1} D & G_{2}\end{array}\right]$. We now use the algorithm in Section 4 employing the following values of the state weight matrix $\mathbf{Q}$ and the control input weight matrix $\mathbf{P}$

$$
\begin{aligned}
& Q=\operatorname{diag}\left[\begin{array}{ll}
Q_{1} & Q_{2}
\end{array}\right] \\
& Q_{1}=\operatorname{diag}\left[\begin{array}{llllllll}
10^{5} & 0 & 0 & 0 & 0 & 0 & 0 & 10^{4}
\end{array}\right] \\
& Q_{2}=\operatorname{diag}\left[\begin{array}{ll}
200 & 20
\end{array}\right] \\
& P=\operatorname{diag}\left[\begin{array}{ll}
1 & 1
\end{array}\right]
\end{aligned}
$$

As is seen only deviations around the stationary values (setpoints) for the top and bottom compositions are considered. Rules for the choice of the weighting matrices $\mathbf{Q}$ and $P$ are given in Balchen (1988). We shoose to have one order of mangitude more weight on $x_{1}$ than $x_{8}$ to compensate for the relative difference in numerical values of $x_{1}$ and $x_{8}$. The results for some different structures of the controller are listed below. The index $\gamma$, Equation (26), is used to compare the suboptimal costs in case 1 and 2 with the optimal cost in case 3 .

Case 1. Feedback from $\boldsymbol{x}_{1}$ and $\boldsymbol{x}_{8}$.

In this case the measurement matrix $\mathbf{D}$ is given by

$$
D=\left[\begin{array}{llllllll}
1 & 0 & 0 & 0 & 0 & 0 & 0 & 0 \\
0 & 0 & 0 & 0 & 0 & 0 & 0 & 1
\end{array}\right]
$$

The following subcases are considered

(a) Multivariable PI-controller

$$
\begin{gathered}
G_{1}=\left[\begin{array}{rr}
-137.9 & -27.5 \\
197.8 & -11.2
\end{array}\right] \quad G_{2}=\left[\begin{array}{cc}
-1.1 & -3.6 \\
11.95 & -0.14
\end{array}\right] \\
J(\bar{G})=92541.3 \quad \gamma=1.03
\end{gathered}
$$


(b) Diagonal PI-controller (correct pairing)

$$
\begin{gathered}
G_{1}=\left[\begin{array}{rr}
0 & -20 \cdot 2 \\
239 \cdot 0 & 0
\end{array}\right] \quad G_{2}=\left[\begin{array}{rr}
0 & -3 \cdot 3 \\
10 \cdot 8 & 0
\end{array}\right] \\
J(\bar{G})=97560 \cdot 1 \quad \gamma=1 \cdot 08
\end{gathered}
$$

(c) Diagonal PI-controller (wrong pairing)

$$
\begin{gathered}
G_{1}=\left[\begin{array}{rr}
-334.4 & 0 \\
0 & -11.2
\end{array}\right] \quad G_{2}=\left[\begin{array}{rr}
-34.2 & 0 \\
0 & -1.9
\end{array}\right] \\
J(\bar{G})=130452.4 \quad \gamma=1.45
\end{gathered}
$$

Case 2. Feedback from $x_{1}$ and $x_{8}$ with specified margin of stability.

We specify that the closed loop eignevalues should be located to the left of a line $-\alpha=-0.01$ in the complex plane. The controller structure is the same as in case $1(b)$.

$$
\begin{gathered}
G_{1}=\left[\begin{array}{rr}
0 & -20 \cdot 2 \\
257.6 & 0
\end{array}\right] \quad G_{2}=\left[\begin{array}{rr}
0 & -16 \cdot 1 \\
32 \cdot 3 & 0
\end{array}\right] \\
J(\bar{G})=137578 \cdot 2 \quad \gamma=1.53
\end{gathered}
$$

Case 3. Feedback from all states

For comparison with the above controllers we compute the controller which has feedback from all states (compositions on all trays). The result is found using the above algorithm. It could also have been found by solving the Riccati-equation directly

$$
\begin{gathered}
G_{1}=\left[\begin{array}{rrrrrrrr}
-152 \cdot 2 & -10 \cdot 4 & -1 \cdot 3 & -0.6 & -1 \cdot 2 & -2.4 & -5 \cdot 1 & -22 \cdot 9 \\
212 \cdot 3 & 15 \cdot 1 & 2 \cdot 4 & 0.2 & -0.6 & -1 \cdot 5 & -2 \cdot 9 & -9.3
\end{array}\right] \\
G_{2}=\left[\begin{array}{rr}
-0.6 & -4 \cdot 5 \\
14 \cdot 1 & -0.2
\end{array}\right] \quad J(\bar{G})=89994 \cdot 1 \quad \gamma=1.0
\end{gathered}
$$

The following comments can be made about these results:

Applying restrictions on the controller-structure will generally lead to higher values of the objective functional $\mathbf{J}$. Note that it may in rare situations happen that the optimal controller turns out to have a specific structure.

Imposing a specified stability margin also increases the objective functional The system must act faster.

For case 2 we see that the absolute values of the elements in $G_{2}$ has increased while $G_{1}$ is approximately unchanged compared to case $1(b)$. This means that the integral time $T_{i}$ has decreased while the proportional constant $k_{p}$ is approximately unchanged in both control loops. This is seen from the relations (correct pairing case)

$$
G_{1}=\left[\begin{array}{cc}
0 & k_{p}^{1} \\
k_{p}^{2} & 0
\end{array}\right], \quad G_{2}=\left[\begin{array}{cc}
0 & k_{p}^{1} / T_{i}^{1} \\
k_{p}^{2} / T_{i}^{2} & 0
\end{array}\right]
$$

In Figure (2) we show some results obtained from simulation experiments with the above controllers when a positive step change in the feed flow to the column of $10 \%$ is applied. 
${ }^{8} 8$
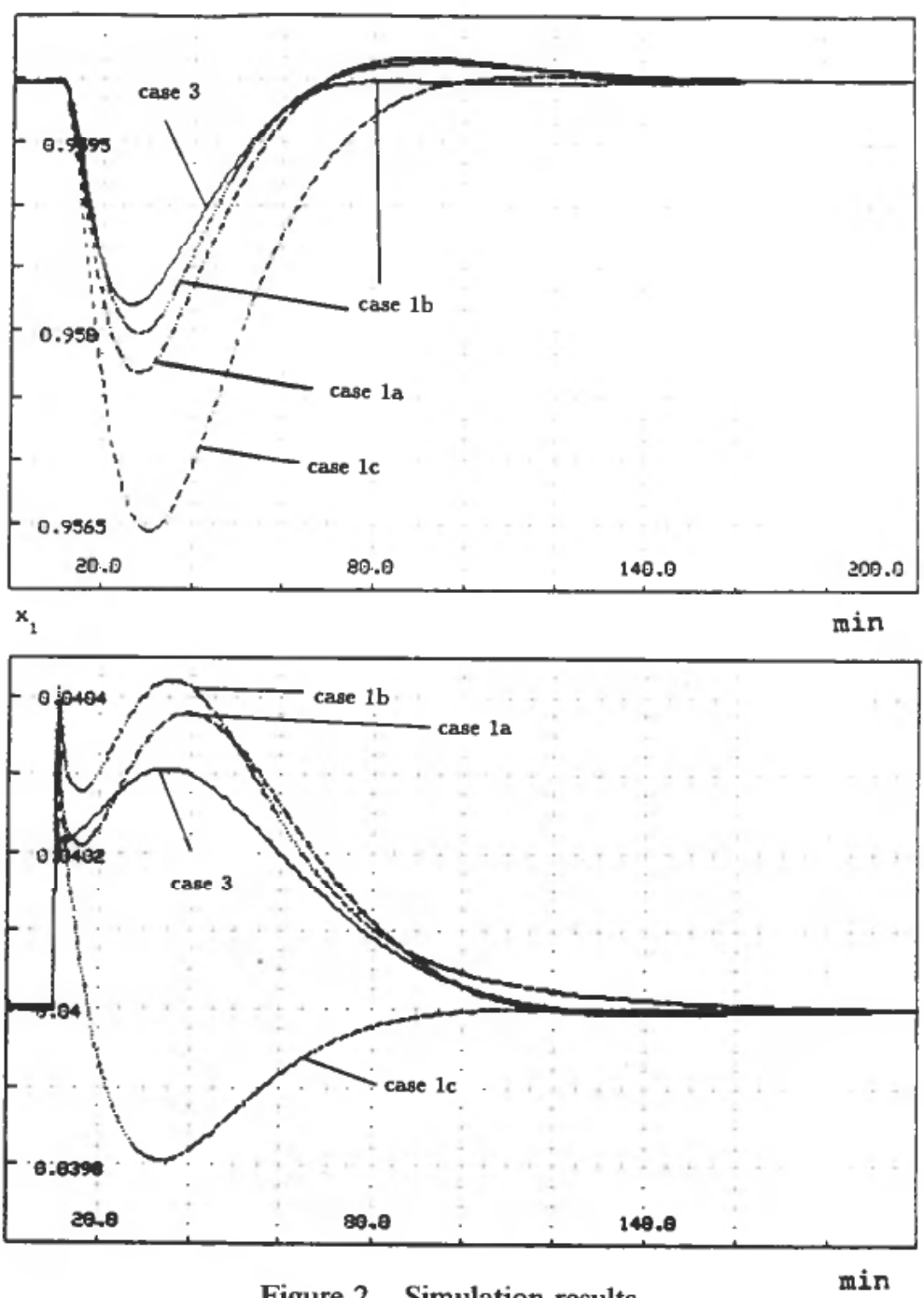

Figure 2. Simulation results.

6.2. Example 2, unstable plant

Consider the open loop unstable plant

$$
\begin{gathered}
A=\left[\begin{array}{rrr}
0 & 1 & 0 \\
0 & 0 & 1 \\
2 & -1 & -2
\end{array}\right] \quad B=\left[\begin{array}{rr}
-1 & 1 \\
1 & 0 \\
0 & 1
\end{array}\right] \\
D=\left[\begin{array}{rrr}
0 & 5 & -1 \\
-1 & -1 & 0
\end{array}\right]
\end{gathered}
$$

The weighting matrices are chosen as

$$
Q=q D^{\mathrm{T}} D=\left[\begin{array}{rrr}
1 & 1 & 0 \\
1 & 26 & -5 \\
0 & -5 & 1
\end{array}\right] \quad P=p\left[\begin{array}{ll}
1 & 0 \\
0 & 1
\end{array}\right]
$$


The designer has the freedom in choosing the scalar weights $q$ and $p$. Starting with the zero control, $q=1$ and $p=1$ the following controllers are found.

(a) Full output controller

$$
G=\left[\begin{array}{rr}
-0.966 & 0.069 \\
0.066 & 1.643
\end{array}\right] J(G)=8.04
$$

(b) Diagonal output controller

$$
G=\left[\begin{array}{rr}
-0.986 & 0 \\
& 1.583
\end{array}\right] J(G)=8.05
$$

(c) Diagonal output controller

$$
G=\left[\begin{array}{rr}
0 & 2 \cdot 189 \\
4 \cdot 131 & 0
\end{array}\right] \quad J(G)=172 \cdot 5
$$

\section{Conclusions}

An algorithm has been developed for the 'optimal' tuning of linear feedback controllers, from the states or from a reduced state vector or from the system outputs, which may have some structure.

If an initial stabilizing controller is available, it has been shown that the application of a modified Newton method for unconstrained optimization facilitiates a relative simple solution to the problem of determining a multivariable controller with possible restrictions to its structure. If an initial stabilizing controller does not exist, constraints to ensure that the final closed loop systems is stable are added to the optimization problem, and a method for constrained optimization is employed.

The algorithm needs only the solution of one matrix Liapunov equation for the determination of the value of the functional to be minimized at each iteration. This is a relatively simple task even for large systems. Furthermore the algorithm is believed to be useful in the design of decentralized controllers where a special structure is desirable.

An interesting feature, in our opinion, is also the fact that the solution will always be stable, at each iteration step in the case of an initially stable plant, and after the final iteration in the case of an initially unstable plant.

This algorithm can in the same manner be used to design stable decentralized estimators. The question of robustness can be solved by implementing an outer optimization loop for the determination of the state weight matrix $\mathbf{Q}$ and control weight matrix $\mathbf{P}$ such that the closed loop system, in addition to minimizing the LQcriterion, also minimizes some robust criterion. One such robust criterion is the structured singular value ( $\mu$ ), Doyle et al. (1982). This problem is solved by Valderhaug, Di Ruscio and Balchen (1990) for the case of no restrictions to the controller structure.

\section{REFERENCES}

Anderson, B. D. O. and Moore, J. B. (1971). Linear Optimal Control (Prentice-Hall). ANDERSON, B. D. O., BOSE, N. K. and JURY, E. I. (1975). Output feedback stabilization and related problems - solution via decision methods. IEEE Trans. on Automatic Control, 20, 53 - 66.

ANDERSON, B. D. O. and SCOTT, R. W. (1977). Output feedback stabilization-solution by algebraic geometry methods. Proc. of the IEEE, 65, 849-861.

Balchen, J. G. and Mummé K. I. (1988). Process Control; Structures and Applications (Van Nostrand Reinhold Company Inc., New York), Chap. 2.11, pp. 63-65.

Bryson, A. E. and Ho, Y. C. (1969). Applied Optimal Control (Blaisdell, New York). 
Bartels, R. H. and Stewart, G. W. (1972). A solution of the equation $A X+X B=C$. Commun. $A C M, 15,820-826$.

CHEN, C. F. and SHIEH, L. S. (1968). A note on expanding $P A+B P=-Q$. IEEE Trans. on Automatic Control, 122-123.

Di Ruscio, D. (1987). Dynamic models for a binary distillation column. Report 87-64-U, Division of Engineering Cybernetics. Norwegian Institute of Technology, N-7034 Trondheim.

Di Ruscio, D. (1988). Solution methods for the Liapunov matrix equation. Internal note, Division of Engineering Cybernetics. Norwegian Institute of Technology, N-7034 Trondheim.

Doyle, J. C., Wall, J. E. and Stein, G. (1982). Performance and robustness analysis for structured uncertainty. Proc. 21th IEEE Conf. Decision and Control, Orlando, Florida, 629-636.

Geromel, J. C. and Bernussou, J. (1979). An algorithm for optimal decentralized regulation of linear quadratic interconnected systems. Automatica, 15, 489-491.

Gill, P. E., Murrag, W., SAUNDERS, M. A. and Wright, M. H. (1986). Users guide for NPSOL. A Fortran package for nonlinear programming. Technical report SOL 86-2. Department of Operational Research, Stanford University, Stanford, CA 94305.

Glub, G. H., NASH, S. and VAN LoAN C. (1979). A Hessenberg-Schur method for the problem $A X+X B=C$. IEEE Trans. on Automatic Control, 24, 909-913.

Kosut, R. L. (1970). Suboptimal control of linear time-invariant systems subject to control structure constraints. $J A C C$, session 31-B.

LeVINE, W. S. and Athans, M. (1970). On the determination of the optimal constant output feedback gain for linear multivariable systems. IEEE Trans. on Automatic Control, 15, 44-48.

SCHITTKOWSKI, K. (1984). NLPQL: A FORTRAN subroutine solving constrained nonlinear programming problems. Working paper, University of Stuttgart, Stuttgart.

Solheim, O. A. (1976). Optimal Control. Tapir. Norweigan Institute of Technology, N-7034 Trondhaim.

Valderhaug, AA., Di Ruscio, D. and Balchen, J. G. (1990). Synthesis of Robust LQcontrollers. 11th IF AC Word Congress, Tallin, Aug. 13-17 1990.

Wolfe, M. A. (1978). Numerical Methods for Unconstrained Optimization (Van Nostrand Reinhold Company Ltd.) 
Distillation column data

Appendix

\section{Assumptions}

Constant molar flows

No vapor holdup (immediate vapor response)

Vapor--Liquid Equilibrium (VLE) and perfect mixing on all trays

Column constants

Number of equilibrium (theoretical) trays excluding the reboiler $N=6$

Feed tray location $N_{F}=3$

Liquid in reboiler $M_{O B}=10 \mathrm{~mol}$

Liquid holdup on each tray $M_{0 i}=5 \mathrm{~mol}, i=1, \ldots, N$

Liquid holdup in accumulator $M_{O D}=10 \mathrm{~mol}$

Relative volatility $\alpha=2.993$

Hydraulic tray constant $K=30_{\text {min }}^{-1}$

Control input to the system

Reflux $R=2 \cdot 0 \frac{\mathrm{mol}}{\mathrm{min}}$

Vapor flow $V=2 \cdot 5_{\text {min }}^{\text {mol }}$

Disturbances to the system

Feed flow $F=1 \cdot 0_{\mathrm{mol}}^{\mathrm{mol}}$

Mole fraction of light component in feed $x_{F}=0.5$

The stationary composition profile is given by

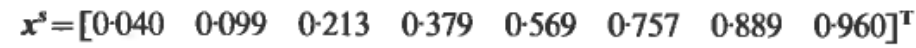

Linearizing the non-linear model around the stationary values gives the following time invariant linear model where the state vector $x=\left(d x_{1}, \ldots, d x_{N+1}\right)^{\mathrm{T}}$, control input vector $u=(d R, d V)^{\mathrm{T}}$ and disturbance input vector $v=\left(d F, d x_{F}\right)^{\mathrm{T}}$ are deviations around stationary values

$$
x=A x+B u+c v
$$

where $\mathrm{A}, \mathrm{B}$ and $\mathrm{C}$ are given below

$$
\mathbf{A}=\left[\begin{array}{rrrrrrrr}
-0.69 & 0.30 & 0 & 0 & 0 & 0 & 0 & 0 \\
1.26 & -1.61 & 0.59 & 0 & 0 & 0 & 0 & 0 \\
0 & 1.02 & -1.31 & 0.59 & 0 & 0 & 0 & 0 \\
0 & 0 & 0.72 & -1.06 & 0.39 & 0 & 0 & 0 \\
0 & 0 & 0 & 0.48 & -0.72 & 0.39 & 0 & 0 \\
0 & 0 & 0 & 0 & 0.32 & -0.63 & 0.39 & 0 \\
0 & 0 & 0 & 0 & 0 & 0.23 & -0.59 & 0.39 \\
0 & 0 & 0 & 0 & 0 & 0 & 0.10 & -0.25
\end{array}\right]
$$

